\title{
Addictive potential of novel treatments for refractory depression and anxiety
}

This article was published in the following Dove Press journal:

Neuropsychiatric Disease and Treatment

\section{Dusan Kolar}

Mood Disorders Research and Treatment Service, Department of Psychiatry, Queen's University, Kingston, Ontario, Canada

Correspondence: Dusan Kolar Department of Psychiatry, Queen's University, Providence Care Hospital, 752 King Street West, Kingston, Ontario K7L 4X3, Canada

Tel +l 6I35444900

Email kolard@providencecare.ca

\begin{abstract}
Treatment-resistant mood disorders and anxiety disorders require intensive treatment, but treatment options should balance benefits and adverse effects or other potential detrimental effects on patients, including the risk of developing prescription medication addiction. Some of the newer treatment modalities for mood and anxiety disorders may have similar properties to benzodiazepines. The goal of this review was to identify the potential for developing dependence on the novel treatment approaches to treatment-resistant depression and refractory anxiety disorders. PubMed, MEDLINE, PsycINFO, Ovid, Cochrane Library, and Google Scholar were searched. Ketamine is effective in improving symptoms of major depressive disorder, but with no sustained benefits. Long-term use of oral or intranasal ketamine formulations may be associated with the risk of developing dependence. Augmentation of stimulant medication is usually effective for residual symptoms of depression, but the effects are usually short lasting and there is a potential for abuse. Synthetic cannabinoids and medicinal cannabis are increasingly being prescribed for a number of medical conditions, including anxiety disorders, without enough evidence about their efficacy and with the risk of patients developing dependence. In summary, benzodiazepines, ketamine, stimulant medications, and cannabinoids have some common characteristics, including short-lasting benefits and the risk of developing prescription medication addiction with longer use. All of these treatments may raise ethical dilemmas about the appropriateness of prescribing these medications in the long run for patients with depression and anxiety disorders.
\end{abstract}

Keywords: treatment-resistant depression, anxiety, addiction, ketamine, cannabinoids

\section{Introduction}

Major depressive disorder and anxiety disorders are the most prevalent mental conditions in psychiatric practice. There has been an increase in the number of patients diagnosed with treatment-resistant depression and anxiety.

These people with treatment refractory conditions may have higher rates of comorbidity with other psychiatric disorders and more general medical comorbidity, inability to work, higher rates of absenteeism, and more frequent hospitalizations and incur higher costs on the health care system.

Treatment-resistant depression and anxiety usually require more intensive treatment and multimodal treatment including different treatment modalities, but medication remains the major treatment component. There are not many new antidepressant medications and clinicians sometimes rely on off-label treatment options. Some of these new treatments have not been sufficiently clinically investigated in terms of safety and possible detrimental effects such as addictive potential. Some of these new agents for refractory depression and anxiety are effective in short-term treatment, but, similarly to benzodiazepines, may be associated with developing tolerance and dependence with 
long-term use. The goal of treatment should not be obtaining instant and short-lasting benefits without taking into account possible negative effects, such as the potential for developing dependence. The aim of good clinical balance is to weigh the benefits and possible negative effects of treatment. Patients may expect from medical professionals instant solutions for their suffering, and this often entails the prescribing of medications with rapid onset of action such as analgesics or benzodiazepine anxiolytics. However, medical professionals should follow ethical principles when prescribing medications and should distinguish agents with the potential to temporarily improve patient symptoms from agents that are genuinely therapeutic.

The patient's interests will be best served by obtaining evidence-based treatments and medications with wellestablished efficacy and safety.

It is well known that benzodiazepines are addictive, but we should not forget that these medications were prescribed for decades as a main treatment option for patients with anxiety disorder. Was there a lack of evidence about the addictive potential of benzodiazepines or was this evidence simply ignored as there were no better alternatives? Currently, a significant number of patients is addicted to benzodiazepines and for many of them benzodiazepines are no longer a sustainable treatment option because of restrictions in prescribing benzodiazepines. Many of these patients were also informed about the increased risk of dementia with longterm use of benzodiazepines, ${ }^{1}$ which is an additional burden on patients. Nine studies have demonstrated this increased risk of dementia in patients on long-term treatment with benzodiazepines, ${ }^{2}$ and this risk is higher in elderly people. ${ }^{3}$

The introduction and easy acceptance of newer treatment options for refractory depression and anxiety, such as ketamine and stimulant medication augmentation in treatment-resistant depression, and synthetic cannabinoids and medicinal cannabis in anxiety disorders and depression, have many characteristics in common with the uncritical prescribing of benzodiazepines for anxiety disorders in the past.

Research studies on the addictive potential of ketamine, stimulant medications, and nabilone are very limited and the literature on this topic is sparse. The goal of this literature review is to explore the available data and provide a critical opinion about using potentially addictive agents in clinical practice.

\section{Method}

Searches of PubMed, MEDLINE, PsycINFO, Ovid, Cochrane Library, and Google Scholar were conducted for placebo-controlled randomized clinical trials, meta-analyses, non-randomized controlled studies, naturalistic studies, case reports, and treatment guidelines published in the past 10 years (from 2008 to $2017 / 2018$ ) on the potential for abuse and dependence of new and not well-investigated medications in the treatment of refractory depression and refractory anxiety disorders. As the Diagnostic and Statistical Manual of Mental Disorders, Fifth Edition (DSM-5) classification was used, post-traumatic stress disorder (PTSD) and obsessivecompulsive disorder were excluded from the category of anxiety disorders and therefore new agents in treating these conditions, besides nabilone, will not be included. Nabilone will be included simply because the off-label use of this medication for anxiety, insomnia, and depression started after the publication of studies on the use of nabilone in PTSD-associated nightmares. This review will not include other common augmentation strategies in treatment-resistant depression, such as atypical antipsychotics and other newer medications such as pramipexole, as the focus of this review is only medication with potential addictive potential.

\section{Ketamine in the treatment of major depressive disorder}

Ketamine is a non-selective $N$-methyl-D-aspartate (NMDA) receptor antagonist. Ketamine has both opiate and stimulant effects. It is a strong promoter of dopamine turnover and ketamine's monoaminergic properties are similar to those of cocaine or amphetamine. Ketamine also has mu-opioid receptor properties. ${ }^{4}$

Ketamine was used as an anesthetic agent primarily in pediatric surgery and veterinary medicine. Pharmacologically, ketamine can be classed as a dissociative anesthetic medication, hallucinogen, and psychotomimetic drug. Ketamine is also well known as a recreational drug because of its psychedelic and dissociative effects.

More recently, ketamine has been used for treating depressive symptoms refractory to other treatments. Clinical trials of ketamine in patients with treatment-resistant depression demonstrated that it has rapid antidepressant effects within 2-4 h, but these benefits of ketamine infusions are short lived, being sustained for only 4-7 days. ${ }^{5}$

A total of 7 trials with 147 ketamine-treated participants showed that ketamine produced a rapid but transient antidepressant effect, accompanied by brief psychomimetic and dissociative effects. ${ }^{6}$

Ketamine is effective in the rapid treatment of unipolar and bipolar depression, both as monotherapy and in combination with other medications. ${ }^{7,8}$ 
Kellner and colleagues reported a series of severely depressed patients, some of whom were suicidal, who received ketamine infusions before being referred for electroconvulsive therapy. These patients had either no antidepressant effects or transient antidepressant benefit from ketamine with unpleasant side effects, mostly dissociative effects. ${ }^{9}$ The authors concluded that evidence-based treatments, including electroconvulsive therapy, should be offered before experimental and unproven treatment approaches.

A meta-analysis which included 7 randomized controlled trials (RCTs) with small sample size reported no serious adverse events in short-term treatment. The authors concluded that the potential for misuse is negligible, although this statement did not come from the results of any of studies included in this meta-analysis. ${ }^{8}$

The most common adverse events after ketamine infusion are dizziness, blurred vision, headache, nausea or vomiting, dry mouth, poor coordination, poor concentration, and restlessness, and these side effects are usually short lasting. About $17 \%$ of patients in one study developed significant dissociative symptoms. ${ }^{10}$ Cardiovascular adverse events were reported, requiring a higher dose of antihypertensive medication, and caution is needed in patients with cardiovascular conditions. ${ }^{7}$ Side effects during and after each ketamine infusion were generally mild in research studies and ketamine caused minimal positive psychotic symptoms. ${ }^{11}$

The potential for abuse and other safety concerns associated with ketamine mean that caution is required when applying ketamine outside the research setting. ${ }^{10}$

Concerns over cognitive impairment, cystitis, liability for abuse, and a potential increase in iatrogenic ketamine disorder related to more frequent use of ketamine suggest that the use of ketamine should be restricted until additional research data are available. ${ }^{12}$

The main concern about the clinical application of ketamine trials is the conclusion that treatment response is short lasting. ${ }^{8}$ In a 4 week placebo-controlled study, the efficacy and safety of an intravenous single dose of ketamine as augmentation of escitalopram in major depressive disorder were examined. ${ }^{13}$ This augmentation strategy was safe and effective in speeding up oral antidepressant efficacy. Effects lasted for 2 weeks, but this study does not provide evidence of sustained benefits as continuous therapy with escitalopram served as a maintenance treatment after discontinuing ketamine. ${ }^{13}$

Another study which failed to demonstrate any longlasting effects of ketamine was an RCT which included 47 patients with treatment-resistant depression. At day 7 after stopping ketamine infusions, only 21 patients still met the criteria for response. ${ }^{10}$ About $89 \%$ of patients relapsed, on average 19 days after receiving 6 infusions of ketamine. ${ }^{11}$

This research evidence of the lack of sustained benefits of ketamine is consistent with clinical experience of short-lived therapeutic effects. The only study demonstrating some sustained benefits of intranasal esketamine is an RCT published by the Janssen Research and Development team. ${ }^{14}$ This study included 67 patients with treatment-resistant depression. Among 22 participants in the follow-up phase, only 10 had improvements in depressive symptoms that persisted up to 2 months after stopping esketamine. ${ }^{14}$ This small sample size in the follow-up phase and a small percentage of participants maintaining benefits do not provide convincing evidence of sustained therapeutic benefits of ketamine.

As the benefits of ketamine are short lasting, ongoing treatment with ketamine would be needed for a long time, similarly to selective serotonin reuptake inhibitors, serotonin-norepinephrine reuptake inhibitors, and other antidepressants. Such regular treatment with ketamine would make it the first time in the history of medicine that an anesthesia medication for short-term use was used for the long-term treatment of a chronic condition, without enough knowledge about the possible consequences and risks of this prolonged therapy. ${ }^{15}$

The literature on using oral and intranasal ketamine formulations is sparse, but there are established ketamine clinics around the world which provide off-label ketamine to patients for extended periods. As ketamine is a well-known recreational drug that can lead to ketamine dependence, treating depression with oral or intranasal ketamine formulations may increase the risk of developing dependence over an extended period, despite the use of much lower doses. There is no available research on the addictive potential of ketamine used in the treatment of depression.

At the current level of knowledge, we can only hypothesize that long-term use of ketamine in the treatment of depression may result, as in drug addicts, in patients developing tolerance, dependence, drug craving, and withdrawal symptoms.

There is some research evidence of neurotoxicity in animals, ${ }^{6}$ and this concern should not be neglected in considering long-term ketamine treatment trials. There is research evidence of abnormalities of white matter in bilateral frontal and left temporoparietal regions following chronic ketamine use. ${ }^{16}$ Detrimental effects of ketamine are evident when used in uncontrolled circumstances.

Recreational ketamine users may develop memory deficits, delusions, hallucinations, ulcerative cystitis, elevated 
liver enzymes, and biliary tract dilatation in the absence of an obstructing lesion. ${ }^{17}$

A significant number of ketamine abusers developed dependence after regular use for 1 year, and $53.5 \%$ of these abusers reported withdrawal symptoms including fatigue, excessive yawning, aggressive or hostile behavior, anger, irritability, and depression. ${ }^{18}$

Ketamine is not recommended as a treatment option for treatment-resistant depression in any treatment guidelines. The Canadian Network for Mood and Anxiety Treatments (CANMAT) guidelines for the management of adults with major depressive disorder emphasize that ketamine is associated with psychotomimetic side effects and the potential for abuse, and that there are very limited data on its safety and efficacy with longer term use. ${ }^{19}$

It seems that the conclusion of the group of authors from Australia, the UK, the USA, and Canada, in an editorial published in the British Journal of Psychiatry in late 2016, is probably the best recommendation on using ketamine in clinical practice: "Much more needs to be learnt about the maintenance of response and long-term outcome before using ketamine more widely in clinical practice." ${ }^{20}$

\section{Stimulant medication augmentation in treatment-resistant depression}

Augmentation is a common strategy in addressing treatment resistance in depression. Among available augmentation strategies, stimulant augmentation is a treatment with less available evidence compared to other augmentations in treatment-resistant depression. The use of methylphenidate and amphetamines for augmentation is limited as there are no guidelines or expert opinion/consensus on the optimal duration of treatment, stimulant dosage, and treatment goals.

The cognitive-enhancing properties of stimulant medications, increased energy level, and general improvement in morning functioning make stimulant medications more acceptable to patients than atypical antipsychotics. Some clinicians unreasonably prescribe stimulant medications in the belief that they are serving the patients' best interests, although there is no strong evidence for the efficacy of stimulant augmentation. ${ }^{21}$ Stimulant augmentation in the treatment of major depressive disorder is a third-line treatment option with a level 3 of evidence in the CANMAT guidelines for Major Depressive Disorder. ${ }^{19}$

Some researchers have tried to demonstrate that psychostimulants may have overall antidepressant effects beyond augmentation, such as in an Australian open study from 2013. Stimulants, including methylphenidate and dextroamphetamine administered as augmentation, were very effective in treating melancholic symptoms for $20 \%$ of patients among the group of 50 patients with unipolar or bipolar depression. For $50 \%$ of patients in each group, stimulants were "somewhat effective" and for 30\% ineffective. ${ }^{22}$

Corp and colleagues carried out a literature review on using stimulants and stimulant alternatives in treating depression and concluded that modafinil and armodafinil are effective treatments for treatment-resistant unipolar and bipolar depression. ${ }^{23}$ Data from randomized clinical trials on methylphenidate and amphetamines are too limited to support the use of stimulants as a first-line augmenting strategy for depression, with the exception of one RCT demonstrating the effects of lisdexamfetamine. ${ }^{23}$ There is limited evidence on the efficacy of lisdexamfetamine in improving executive dysfunctions and depressive symptoms in patients with mild major depressive disorder. ${ }^{24}$

Stimulant medications could address certain residual depressive symptoms, including decreased energy, lack of concentration, decreased alertness, and daytime sleepiness, but unfortunately these benefits are not sustained and patients lose all of these positive effects of stimulants after medications are discontinued. However, it is not uncommon to see patients using stimulants continuously for several years or even longer. ${ }^{25}$ As a result of this long-term use, the patients may develop dependence on stimulants, particularly amphetamines. There is no research evidence on long-term use of stimulant medications in treatment-resistant depression, but in clinical practice there are patients with depression receiving stimulants for a few years or longer.

There is little research evidence about the potential for addiction of stimulant medications, probably because these medications are mostly used in the treatment of attentiondeficit hyperactivity disorder (ADHD) in children and adolescents, and the prevailing hypothesis in child psychiatry is that treating ADHD will prevent the development of substance use disorder. On the other hand, trials on the use of stimulant medication (lisdexamfetamine) in treating cocaine dependence ${ }^{26}$ and methylphenidate in the treatment of amphetamine/metamphetamine dependence ${ }^{27}$ may imply the potential of prescription stimulant medications to be used as substitution therapy.

There is concern over the non-medical use and misuse of stimulant medications in individuals without ADHD, particularly the misuse of short-acting agents, ${ }^{28,29}$ and the potential for abuse is equated with illicit central nervous system stimulants such as amphetamine and cocaine. ${ }^{30}$ However, there is no research evidence for patients being dependent on 
prescription stimulant medications in the context of long-term treatment. A group of authors from France emphasized that the risk factor for dependence on methylphenidate in children who receive normal doses is the duration of treatment. ${ }^{31}$

In the case of stimulant augmentation in major depressive disorder, discontinuing stimulants after long-term use could cause sudden and significant declines in energy level, motivation, and cognitive performance, as well as high anxiety and restlessness. These types of withdrawal symptoms provide the best evidence of dependence on stimulants.

However, if Parker et al concept of using stimulants as antidepressants ${ }^{22}$ became widely accepted, then individuals with depression could be taking stimulant medications for most of their life, similarly to patients with ADHD.

\section{Medicinal cannabis and synthetic cannabinoids in anxiety and depression}

Medicinal cannabis is considered nowadays as a treatment for various medical conditions such parkinsonism, hepatitis $\mathrm{C}$, pain management in cancer, spasticity in patients with multiple sclerosis, neuropathic pain, refractory epilepsy, glaucoma, and some psychiatric conditions (anxiety and depression).

Cannabis and synthetic cannabinoids exert their effects via the endocannabinoid system. Cannabinoid receptors (CB1 and $\mathrm{CB} 2$ ) are present in the central nervous system and throughout the body. They are activated by cannabis, synthetic cannabinoids, or endogenous endocannabinoids. ${ }^{32}$ This presumed mechanism of action is an explanation for possible the therapeutic effects of medicinal cannabis. However, it is still difficult to understand the wide range of therapeutic indications for medicinal cannabis.

It also seems that public approval is driving the medicinal cannabis legalization process without going through the standardized procedures for new drug development and approval. $^{33}$

Medicinal marijuana and recreationally used marijuana differ in how the drug is used in terms of the amount and goals of ingestion, although there is a significant overlap between medicinal users and recreational users. ${ }^{33}$

Cannabis is the most widely used illicit drug in the USA. Among the 20 million Americans who used marijuana in 2013 , it was found that up to $9 \%$ of users may become dependent and this number goes up to $17 \%$ if marijuana use is started during adolescence. ${ }^{34}$ Long-term and regular use of cannabis for medical conditions could be associated with an even higher rate of cannabis dependence and this medical use is particularly problematic in psychiatric conditions such as anxiety disorders.

The results of a systematic review and meta-analysis on the use of cannabinoids in medicine did not provide any evidence for the efficacy of cannabis and cannabinoid drugs in treating anxiety. There is some evidence of efficacy in the treatment of chronic pain and spasticity. ${ }^{35}$

The evidence to support using cannabis in the treatment of anxiety disorders and mood disorders is very limited and includes a few single-dose studies. ${ }^{36}$ On the other hand, cannabis is associated with adverse effects including increased anxiety, psychosis, cognitive impairment, and addiction, which pose significant limitations to using cannabis as a treatment in psychiatry. ${ }^{36}$

The unjustified belief that medicinal cannabis may have anxiolytic effects comes from reports about decreased anxiety levels after taking cannabis. This transitory decrease in anxiety level after a single dose, with a subsequent increase in baseline anxiety, is the best evidence that medicinal marijuana is not a sustainable treatment option for anxiety disorders. Psychiatric patients in general and patients with a history of substance use disorders are not candidates for medicinal cannabis at all. ${ }^{37}$

Frequent cannabis users have a higher prevalence of anxiety disorders, but there is not enough evidence of increased risk for developing long-lasting anxiety disorders in cannabis users. ${ }^{38}$

There are single reports of marijuana being used successfully to treat PTSD symptoms. ${ }^{39}$ This is somewhat surprising because substance abuse, including cannabis use disorder, is a common psychiatric comorbidity/complication in patients with PTSD, but now we have the situation where cannabis is being used for PTSD treatment.

A synthetic cannabinoid, nabilone, has been used lately for treating nightmares associated with severe PTSD. However, nabilone has been approved only for the treatment of cancer therapy-induced nausea.

An open-label clinical trial on using nabilone in 47 patients diagnosed with PTSD demonstrated that adjunctive use of nabilone (Cesamet ${ }^{\mathrm{TM}}$ ) resulted in a significant reduction in nightmare intensity and improvement in the quality of sleep. ${ }^{40}$ In 2015, a group of Canadian military psychiatrists published a randomized placebo-controlled study on the efficacy of nabilone in the treatment of PTSD-associated nightmares. They found a significant reduction in nightmares in the population of military patients with PTSD. The major limitation of this study is the small sample size of only 10 patients. $^{41}$ 
There have been no studies on the addictive potential of nabilone. There is only one study, published in 2010, saying that reports of nabilone abuse are rare, but the authors recommended a follow-up of patients, including assessment of tolerance and dependence. ${ }^{42}$

Nabilone is currently a commonly prescribed medication for nightmares associated with various clinical conditions, not only PTSD, and long-term use of nabilone is, unfortunately, not uncommon. There is a tendency for nabilone or even medicinal cannabis to be prescribed for patients with anxiety, insomnia, and depression, without research evidence of therapeutic effects and with unknown long-term consequences. ${ }^{37}$

Since cannabis induces a sense of euphoria, it has been reported that many people begin cannabis use during depressive episodes. ${ }^{36}$ However, it would be a pitfall for clinicians to prescribe medicinal cannabis for patients with depression as a transitory experience of euphoria is not a therapeutic effect.

\section{Discussion}

Benzodiazepines, ketamine, stimulant medications, and cannabinoids have some common characteristics which are elaborated on in this paper. All of these agents may help patients with mood and anxiety disorder symptoms to a certain degree, but it is very important to make a distinction between agents that may make patients feel better and agents that are genuinely therapeutic.

These agents could alleviate patients' suffering in the short run, but their longer use may have undesirable effects, including the possibility of developing tolerance and dependence, and the potential for toxicity with long-term administration.

We learned this lesson with benzodiazepines, but this knowledge does not prevent many physicians from prescribing or even overprescribing off-label treatment options such as stimulant medications and ketamine for patients with major depressive disorder, or synthetic cannabinoids and medicinal cannabis for patients with anxiety disorders. Benzodiazepines were uncritically overprescribed for decades as a main treatment option for anxiety disorders, while at present there is a strong recommendation in many countries to avoid prescribing benzodiazepines whenever possible.

Ketamine has rapid antidepressant effects, but these effects are short lived and usually last from only a few days to a maximum of 7 days. ${ }^{5}$ Therefore, if clinicians are aiming to treat major depressive disorder with ketamine, regular use is needed to achieve a therapeutic effect. However, prescribing oral or intranasal formulations of ketamine, a well-known recreational drug, can easily foster an addiction to prescription medication. Ethical dilemmas about prescribing benzodiazepines and ketamine are very similar with regard to their potential for the development of dependence.

Generations of medical students and psychiatrists were taught about the detrimental effects of cannabis on mood disorders and anxiety disorders, but we are now witnessing the process of the gradual introduction of medicinal marijuana in treating various medical conditions and, possibly, in treating psychiatric disorders. Is this due to a change in the psychiatric paradigm or temporary confusion in psychiatric practice influenced by the lack of new and effective evidencebased treatments in psychiatry ${ }^{43}$

Stimulant augmentation in the treatment of major depressive disorder is a third-line (level 3 of evidence) treatment option/augmentation strategy in the Canadian guidelines for mood and anxiety disorders ${ }^{19}$ for cases of very refractory depression. Stimulant augmentation is supposed to help patients with residual symptoms to reach remission. However, patients may carry on receiving continuously stimulant medications for a few years, or usually longer, in spite of gaining only some partial benefits from this add-on treatment. ${ }^{21}$ Augmentation with stimulant medications should not be a long-term treatment option.

Clinicians should refrain from prescribing all of these offlabel treatment options in regular clinic practice until more research evidence on their efficacy and safety is available. The risk of dependence with regular use of oral or intranasal ketamine, long-term use of stimulant medication, and synthetic cannabinoids and medicinal cannabis is significant. Psychiatrists should recall negative previous experiences with benzodiazepines to avoid potential pitfalls in clinical practice using new medications with similar properties.

\section{Disclosure}

The author reports no conflicts of interest in this work.

\section{References}

1. Billioti de Gage S, Moride Y, Ducruet T, et al. Benzodiazepine use and risk of Alzheimer's disease: case-control study. BMJ. 2014;9:349.

2. Billioti de Gage S, Pariente A, Bégaud B. Is there really a link between benzodiazepine use and the risk of dementia? Expert Opin Drug Saf. 2015;14(5):733-747.

3. Wu CS, Wang SC, Chang IS, Lin KM. The association between dementia and long-term use of benzodiazepine in the elderly: nested casecontrol study using claims data. Am J Geriatr Psychiatry. 2009;17(7): 614-620.

4. Schatzberg AF. A word to the wise about ketamine. Am J Psychiatry. 2014;171(3):262-264.

5. Williams NR, Schatzberg AF. NMDA antagonist treatment of depression. Curr Opin Neurobiol. 2015;36:112-117.

6. Newport DJ, Carpenter LL, McDonald WM, et al. Ketamine and other NMDA antagonists: early clinical trials and possible mechanisms in depression. Am J Psychiatry. 2015;172(10):950-966. 
7. Fond G, Loundou A, Rabu C, et al. Ketamine administration in depressive disorders: a systematic review and meta-analysis. Psychopharmacology (Berl). 2014;231(18):3663-3676.

8. McGirr A, Berlim M, Bond D, Fleck M, Yatham L, Lam R. A systematic review and meta-analysis of randomized, double-blind, placebocontrolled trials of ketamine in the rapid treatment of major depressive episodes. Psychol Med. 2015;45(4):693-704.

9. Kellner CH, Greenberg RM, Ahle GM, Liebman LS. Electroconvulsive therapy is a standard treatment; ketamine is not (yet). Am J Psychiatry 2014;171(7):796.

10. Murrough JW, Iosifescu DV, Chang LC, et al. Antidepressant efficacy of ketamine in treatment resistant major depression: a two-site randomized controlled trial. Am J Psychiatry. 2013;170(10):1134-1142.

11. aan het Rot M, Collins KA, Murrough JW, et al. Safety and efficacy of repeated-dose intravenous ketamine for treatment-resistant depression. Biol Psychiatry. 2010;67(2):139-145.

12. Wilkinson ST, Sanacora G. Considerations on the off-label use of ketamine as a treatment for mood disorders. JAMA. 2017;318(9):793-794.

13. Hu YD, Xiang YT, Fang JX, et al. Single i.v. ketamine augmentation of newly initiated escitalopram for major depression: results from a randomized, placebo-controlled 4-week study. Psychol Med. 2016;46(3): 623-635.

14. Daly EJ, Singh JB, Fedgchin M, et al. Efficacy and safety of intranasal esketamine adjunctive to oral antidepressant therapy in treatmentresistant depression: a randomized clinical trial. JAMA Psychiatry. 2018; 75(2):139-148.

15. Freedman R. Further investigation of ketamine. Am J Psychiatry. 2016; 173(8):761-762

16. Liao Y, Tang J, Ma M, et al. Frontal white matter abnormalities following chronic ketamine use: a diffusion tensor imaging study. Brain. 2010; 133:2115-2122.

17. Niesters M, Martini C, Dahan A. Ketamine for chronic pain: risks and benefits. Br J Clin Pharmacol. 2014;77(2):357-367.

18. Li JH, Vicknasingam B, Cheung YW, et al. To use or not to use: an update on licit and illicit ketamine use. Subst Abuse Rehabil. 2011;2:11-20.

19. Kennedy SH, Lam RW, McIntyre RS, et al. Canadian Network for Mood and Anxiety Treatments (CANMAT) 2016 Clinical Guidelines for the Management of Adults with Major Depressive Disorder: Section 3 Pharmacological Treatments. Can J Psychiatry. 2016;61(9):540-560.

20. Mahli GS, Byrow Y, Cassidy F, et al. Ketamine: stimulating antidepressant treatment? BJPsych Open. 2016;2(3):e5-e9.

21. Kolar D. Emerging treatments in the management of patients with mood and anxiety disorders - practical and ethical considerations. Oral presentation, XXXIVth International Congress on Law and Mental Health; July 12-17, 2015; Vienna, Austria.

22. Parker G, Brotchie H, McClure G, Fletcher K. Psychostimulants for managing unipolar and bipolar treatment-resistant melancholic depression: a medium-term evaluation of cost benefits. J Affect Disord. 2013;151:360-364.

23. Corp SA, Gitlin MJ, Altshuler LL. A review of the use of stimulants and stimulant alternatives in treating bipolar depression and major depressive disorder. J Clin Psychiatry. 2014;75(9):1010-1018.

24. Madhoo M, Keefe R, Roth RM, et al. Lisdexamfetamine dimesylate augmentation in adults with persistent executive dysfunction after partial or full remission of major depressive disorder. Neuropsychopharmacology. 2014;39(6):1388-1398.
25. Kolar D. Alertness in patients with treatment resistant depression. Kingston, Ontario: Queen's University Research Initiation Grant; 2013.

26. Mooney ME, Herin DV, Specker S, Babb D, Levin FR, Brabowski J. Pilot study of the effects of lisdexamfetamine on cocaine use: a randomized, double-blind, placebo-controlled trial. Drug Alcohol Depend. 2015;153:94-103.

27. Miles WS, Sheridan J, Russell B, et al. Extended-release methylphenidate for treatment of amphetamine/methamphetamine dependence: a randomized, double-blind, placebo-controlled trial. Addiction. 2013; 108(7):1279-1286.

28. Weyandt LL, Oster DR, Marraccini ME, et al. Pharmacological interventions for adolescents and adults with ADHD: stimulant and nonstimulant medications and misuse of prescription stimulants. Psychol Res Behav Manag. 2014;7:223-249.

29. Rabiner DL. Stimulant prescription cautions: addressing misuse, diversion and malingering. Curr Psychiatry Rep. 2013;15(7):375.

30. Benson K, Flory K, Humphreys KL, Lee SS. Misuse of stimulant medication among college students: a comprehensive review and metaanalysis. Clin Child Fam Psychol Rev. 2015;18:50-76.

31. Chéron-Blumel A, Victorri-Vigneau C, Hardouin J, et al. Methylphenidate prescription: important recommendations to mitigate dependence. Neuropsychiatrie de l'Enfance et de l'Adolescence. 2012; 60(5):S254

32. Pacher P, Bátkai S, Kunos G. The endocannabinoid system as an emerging target of pharmacotherapy. Pharmacol Rev. 2006;58(3):389-462.

33. Botstwick JM. Blurred boundaries: the therapeutics and politics of medical marijuana. Mayo Clinic Proc. 2012;87:172-186.

34. Shuman J, Hill J, Saxon AJ, Levin FR. Controversies in marijuana legalization: taking the high road. MD Conference Express. 2015;15(11): $12-14$.

35. Whiting PF, Wolff RF, Deshpande S, et al. Cannabinoids for medical use: a systematic review and meta-analysis. JAMA. 2015;313(24): 2456-2473.

36. Turna J, Patterson B, Van Amerigan M. Is cannabis treatment for anxiety, mood, and related disorders ready for prime time? Depress Anxiety. 2017;34(11):1006-1017.

37. Kolar D, Kolar MV. Critical review of available treatment options for refractory depression and anxiety - clinical and ethical dilemmas. Med Pregl. 2016;69(5-6):171-176.

38. Crippa JA, Zuardi AW, Martín-Santos R, et al. Cannabis and anxiety: a critical review of the evidence. Hum Psychopharm Clin. 2009;24: 515-523.

39. Papini S, Sullivan GM, Hien DA, Shvil E, Neria Y. Toward a translational approach to targeting the endocannabinoid system in posttraumatic stress disorder: a critical review of preclinical research. Biol Psychol. 2015;104: $8-18$.

40. Fraser GA. The use of a synthetic cannabinoid in the management of treatment-resistant nightmares in posttraumatic stress disorder (PTSD). CNS Neurosci Ther. 2009;15(1):84-88.

41. Jelty R, Heber A, Fraser G, Boisvert D. The efficacy of nabilone, a synthetic cannabinoid, in the treatment of PTSD-associated nightmares: a preliminary randomized, double-blind, placebo-controlled cross-over design study. Psychoneuroendocrinology. 2015;51:585-588.

42. Ware MA, St Arnaud-Tempe E. The abuse potential of the synthetic cannabinoid nabilone. Addiction. 2010;105(3):494-503.

43. Kolar D. Controversies in psychiatry. Synergy. 2017;24(13):7-9.

Neuropsychiatric Disease and Treatment

\section{Publish your work in this journal}

Neuropsychiatric Disease and Treatment is an international, peerreviewed journal of clinical therapeutics and pharmacology focusing on concise rapid reporting of clinical or pre-clinical studies on a range of neuropsychiatric and neurological disorders. This journal is indexed on PubMed Central, the 'PsycINFO' database and CAS,

and is the official journal of The International Neuropsychiatric Association (INA). The manuscript management system is completely online and includes a very quick and fair peer-review system, which is all easy to use. Visit http://www.dovepress.com/testimonials.php to read real quotes from published authors. 